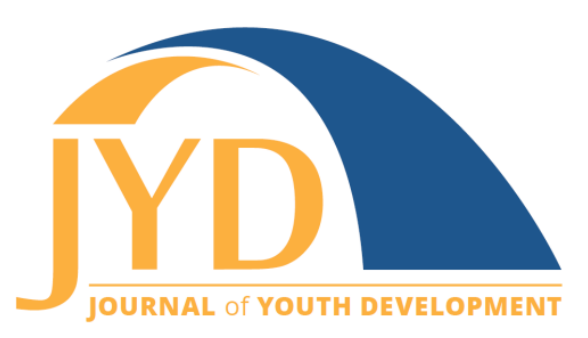

http://jyd. pitt. edu/ | Vol. 14 Issue 3 DOI 10.5195/jyd.2019.731 | ISSN 2325-4017 (online)

\title{
Removing Barriers to Treatment: Evaluation of a Youth Suicide Prevention Program
}

\author{
Franci Crepeau-Hobson \\ University of Colorado, Denver \\ franci.crepeau-hobson@ucdenver.edu \\ James Estes \\ Cherry Creek School District \\ jestes@cherrycreekschools.org
}

\begin{abstract}
This study examined the effectiveness of a suicide prevention program that provides free and accessible counseling services to suicidal youth. Children and adolescents who underwent a suicide risk assessment at a Colorado school $(\mathrm{n}=99)$ were referred for counseling through the Second Wind Fund (SWF), a nonprofit organization dedicated to the prevention of youth suicide. Each of these clients presented with at least 1 barrier to treatment such as no or inadequate insurance or lack of transportation. Through SWF, clients received up to 12 counseling sessions at no charge. Clients ranged from 8 to 18 years of age. Of the 99 clients, 93 completed the Suicide Ideation Questionnaire-Junior (SIQ-Jr) at the beginning and end of treatment. Results indicated that SIQ-Jr scores were significantly lower at the end of treatment. Follow-up surveys were sent to referral sources at the end of the subsequent school year to examine longer-term effectiveness of SWF services. The majority of clients did not require any additional intervention and none went on to die by suicide. Study results indicate that the removal of financial and social barriers can increase access to and participation in treatment and reduce suicidal thoughts and behaviors. Implications for practice, including suicide risk assessment and outside referrals are discussed.
\end{abstract}

Key words: youth suicide, suicide treatment, barriers to treatment

\section{Introduction}

According to the Centers for Disease Control (CDC), suicide is the second leading cause of death among American youth aged 10 to 14 years and 15 to 24 years (Heron, 2016). In addition to deaths by suicide, suicidal ideation and attempts also appear to be major concerns.

(c) $\mathbf{E Y}$ New articles in this journal are licensed under a Creative Commons Attribution 4.0 License. This journal is published by the University Library System, University of Pittsburgh and is cosponsored by the University of Pittsburgh Press. The Journal of Youth Development is the official peer-reviewed publication of the National Association of Extension 4-H Agents and the National AfterSchool Association. 


\section{Removing Barriers to Treatment for Suicidal Youth}

For example, data from the 2015 National Youth Risk Behavior Survey (YRBS), the largest survey of U.S. youth that monitors health risk behaviors, suggest that approximately $17 \%$ of all American high school students report having seriously considered committing suicide and more than $8 \%$ actually have attempted suicide in the previous 12 months (Kann et al., 2016). Gender differences in deaths by suicide have been noted in youth with males dying by suicide at much higher rates than females (CDC, 2017). However, some research suggests that younger females (15 years and under) have higher rates than males (Guerra \& Vasiliadis, 2016).

There are a number of factors that are related to increased risk for suicide in youth. The most powerful of these are biological and psychopathology/psychological risk factors such as depression (Fried, Williams, Cabral, \& Hacker, 2012; Verona, \& Shabnam, 2011); previous suicide attempt (Fried et al., 2012); substance use (Cherpitel, Borges, \& Wilcox, 2004; Kelly, Cornelius, \& Lynch, 2002; Verona, \& Shabnam, 2011); and attention-deficit/hyperactivity disorder (Impey \& Heun, 2012).

Although a variety of psychosocial interventions known to be effective in treating youth who are depressed and/or suicidal are available (Ougrin, Tranah, Stahl, Moran, \& Asarnow, 2015), most adolescents suffering from depression do not receive treatment (Asarnow, Tompson, \& Berk, 2005; Cummings \& Druss, 2011). This is also true for most suicidal youth (Farand, Renaud, \& Chagnon, 2004; Freedenthal, 2007). Among children 6 to 17 years old in the National Survey of American Families estimated to have need of mental health services, $79 \%$ had not used these services in the past 12 months (Kataoka, Zhang, \& Wells, 2002). More recent studies still indicate less than half of youth reporting current serious suicidal ideation, depression, substance problems, or functional impairment are receiving treatment (Gould, et al., 2009; Wu, Katic, Liu, Fan, \& Fuller, 2010). For those who do receive treatment, it usually is not accessed for at least a year following the onset of mental health symptoms (Wang, Bergland, Olfson, \& Kessler, 2004).

Prior studies have shown multiple demographic trends among suicidal youth who receive mental health services (Michelmore \& Hindley, 2012; Chu, Hsieh, \& Tokars, 2011; Kataoka, Stein, Nadeem, \& Wong, 2007; Kataoka et al., 2002; Wu et al., 2010). One factor of significance is the racial/ethnic identity of the child. White adolescent suicide attempters were significantly more likely to receive inpatient or outpatient services (Wu et al., 2010), while being in an ethnic minority group was independently associated with not seeking professional help and with a lack of receipt of counseling in suicidal adolescents (Michelmore \& Hindley, 2012). Specifically, it has been found that a higher percentage of Hispanic children in need (88\%) than white children in need $(76 \%)$ did not receive care (Kataoka et al., 2002) and following a crisis intervention at 


\section{Removing Barriers to Treatment for Suicidal Youth}

school, Latinos were less likely than non-Latinos to have seen a community mental health provider (43\% vs. 73\%; Kataoka et al., 2007). Furthermore, 35.7\% of Asian Americans with prior suicide attempts had never sought any help in their lifetime and Asian Americans with suicidal ideation were found to be less likely to seek and perceive a need for help than their Latino counterparts (Chu et al., 2011). However, it should be noted that foreign-born adolescents of various ethnicities were found to be more likely to receive mental health treatment in inpatient settings (Wu et al., 2010).

Differences in service access have also been noted by gender. Research indicates that males who died by suicide were significantly less likely to have accessed mental health services in the year prior to their deaths (Farand et al., 2004; Guerra \& Vasiliadis, 2016). This may contribute to the significantly higher rates of suicides in males.

Prior service utilization research has focused on a range of factors that influence the receipt of child mental health care. Some of this research has focused on barriers to treatment (Larson et al., 2011, Gould et al., 2009; Kataoka et al., 2007; Moskos, Olson, Halbern, \& Gray, 2007; Owens et al., 2002). Barriers identified include structural barriers such as not knowing where to get help and lack of transportation, barriers related to perceptions of mental health problems, and barriers related to perceptions of mental health services. Access to mental health treatment is also impacted by insurance limitations and the financial cost of treatment (Owens et al., 2002; Moscos et al., 2007). Kataoka et al. (2002) found that a higher percentage of children with public insurance used mental health services (9-13\%) than did the uninsured (4-5\%) and privately insured (5-7\%). Of those children in need of mental health treatment, a higher percentage of uninsured children (87\%) than those with public insurance $(73 \%)$ received no care.

School-based mental health professionals often are the first to recognize the mental health issues of children and youth (Allison, Roeger, \& Abbot, 2008) and thus are central in suicide prevention efforts. Research indicates that school-based suicide prevention and intervention programs can be effective in reducing suicide risk (Crepeau-Hobson, 2013). Schools are also critical in terms of assisting struggling youth and their families in accessing services and intervention, including making referrals to community mental health treatment when schoolbased services are insufficient in meeting the individual's needs (Allison et al., 2008). Research suggests that the majority of youth screened at risk of suicide follow through with referrals for community-based treatment with rates ranging from $70 \%$ (Gould et al., 2009) to about $76 \%$ (Husky, Sheridan, McGuire, \& Olfson, 2011). The findings of these studies indicate suicide risk 


\section{Removing Barriers to Treatment for Suicidal Youth}

assessment strategies within schools can serve as a means of preventing suicide and obtaining mental health services for suicidal youth. However, as noted above, there are a number of financial and social barriers to accessing treatment. This study sought to examine the effectiveness of removing some of these barriers by providing free and easily accessible counseling services to youth at risk for suicide.

\section{Method}

\section{Program}

Second Wind Fund (SWF) is a nonprofit organization in Colorado that provides free therapy to suicidal youth and adolescents who have barriers to accessing treatment. Youth across the entire state of Colorado are referred to SWF services via mental health professionals in schools, hospitals, and other community organizations. In order to qualify for SWF services, the youth must indicate some level of risk for attempting suicide and have a barrier to receiving therapy on their own. Barriers can include a lack of insurance or Medicaid, transportation barriers (unable to get to a therapist's office), financial barriers, and psychosocial barriers (such as the parents are unwilling to seek treatment for their suicidal child). Therapists in the SWF network are carefully screened and monitored and have expertise in child and/or adolescent mental health and significant experience providing evidence-based treatment to children and/or youth at risk for suicide.

Following identification of suicide risk and referral to SWF services, clients are matched with a licensed mental health professional in their geographic region and receive up to 12 counseling sessions free of charge. Consideration of special issues (e.g., sexual minority youth, primary language spoken, etc.) is also given when matching clients to therapists. If transportation is not available, the SWF therapist will travel to the client's school to provide services.

\section{Sample}

The sample consisted of 99 youth referred for counseling through SWF following a suicide risk assessment conducted by a school-based mental health professional at the child's school. On the basis of this assessment, each of these individuals had been deemed at risk of suicide and in need of mental health treatment. 


\section{Removing Barriers to Treatment for Suicidal Youth}

\section{Procedure}

Subsequent to the referral for SWF services, clients were matched with a licensed mental health professional in their geographic region and received up to 12 counseling sessions free of charge. The average time between referral and first session was 3.5 days. The number of counseling sessions provided to the clients in this sample ranged from two to 12 with an average of 8.4 sessions $(S D=3.5)$. Therapists were asked to administer the Suicide Ideation Questionnaire-Junior (SIQ-Jr) or the Suicide Ideation Questionnaire (SIQ; Reynolds, 1987) to each of their clients at the beginning and towards the end of treatment. Some therapists administered one of these questionnaires at mid-treatment as well. Each client's SIQ was sent to SWF staff who entered the scores into an Excel spreadsheet for analysis. The subsequent school year, referring school personnel were emailed a link to an online follow-up survey developed by the researchers to examine longer term effectiveness of SWF counseling.

\section{Measures}

SIQ/SIQ-Jr

The Suicide Ideation Questionnaire (SIQ; Reynolds, 1987) contains 30 items and the SIQ-Jr contains 14 of the same items as the SIQ, as well as one additional item. The SIQ forms are scored on a scale of 0 to 6 as follows:

0 : I never had this thought.

1: I had this thought before but not in the past month.

2: I had this thought about once a month.

3: I had this thought a couple of times a month.

4: I had this thought about once a week.

5: I had this thought a couple of times a week.

6: I had this thought almost every day.

Items are scored in a pathology direction, so that a high score is indicative of numerous suicidal cognitions occurring with significant regularity. A cutoff score was developed to define a level of suicidal ideation that indicates potentially significant suicide risk (Reynolds, 1987). The cutoff score for the SIQ-Jr is 31. The SIQ-JR has high score reliability and validity with a reliability coefficient of .94. Content and construct validity are consistently supported in published clinical studies (e.g., Gutierrez \& Osman, 2009; Reinecke, Du Bois, \& Schultz, 2001). 


\section{Removing Barriers to Treatment for Suicidal Youth}

\section{Post-Therapy Evaluation Survey}

An electronic survey emailed to the school-based referring sources the spring of the subsequent school year included the following four items:

1. When was the last time you had contact with the client?

2. Has the client undergone any suicide risk assessments since the referral for services?

3. Has the client been hospitalized for suicidal/thoughts behaviors since the referral?

4. Has the client attempted suicide since the referral?

\section{Results}

\section{SIQ/SIQ-Jr Results}

Demographic data for a total of 99 SWF clients referred during the 2015-16 school year was collected. Ninety-three of the clients completed a baseline SIQ or SIQ-Jr questionnaire. About $60 \%(n=55)$ of the 93 clients were administered a full SIQ. In an effort to maximize the amount of data that could be analyzed, only the SIQ-Jr items were analyzed for all clients, regardless of which measure was administered. As noted above, the SIQ and SIQ-Jr share 14 items; thus, clients who completed the SIQ had missing data for the one additional SIQ-Jr item.

This sample of 93 clients was between the ages of 8 and 18 years, with a mean age of 14.4 years $(S D=1.99)$. Due to small sample sizes for the youngest and the oldest age groups, children aged 12 and under were grouped together, as were participants aged 17 and older. In the sample, $37 \%$ were male clients and $63 \%$ were female clients. Within this sample, $59 \%$ of the clients were Caucasian, 25\% of the clients were Latino, 3\% were African American, 11\% were of mixed race, and one client was Asian American. One client's race was not reported. Because the sample sizes were so small, African American, Asian American, and mixed race were collapsed into one "Other" variable for the analyses.

Scores for the first administration of the SIQ-Jr items (SIQ-Jr-1) ranged from 2 to 81 with a mean score of $38.2(S D=21.6)$ and a median score of 36.0. A second SIQ/SIQ-Jr (SIQ-Jr-2) was completed by $84 \%$ of the sample $(n=78)$ and $46 \%$ of the sample $(n=43)$ completed a third SIQ/SIQ-Jr (SIQ-Jr-3). The range of time between the administration of SIQ-JR-1 and SIQJR-2 was 14 to 110 days with an average of 46.6 days $(S D=19.8)$. There were an average of 78.8 days between administrations of SIQ-Jr-2 and SIQ-Jr-3 $(S D=46.8)$ and an average of 122 days between administrations of SIQ-Jr-1 and SIQ-Jr-3 (SD = 53.1). The scores for the SIQ-Jr-2 ranged from 0 to 70 and the mean score was $29.6(S D=17.9)$ with a median score of 23.0. 


\section{Removing Barriers to Treatment for Suicidal Youth}

The scores for SIQ-Jr-3 ranged from 0 to 74. The mean score for SIQ-Jr-3 was 24.3 (SD= 17.6) with a median score of 11.5. MANOVA analyses revealed no statistically significant differences on any SIQ-Jr measure based on gender, age, or race/ethnicity. The demographic breakdown for the SIQ-Jr is represented in Table 1.

Despite a large standard deviation in scores, there was only a moderate positive skew of scores and a large enough sample size allowing for a $t$-test to be conducted. A $t$-test was performed to compare the results between the three administrations of the SIQ-Jr. The results demonstrated a statistically significant difference between SIQ-Jr-1 and SIQ-Jr-2, $t(73)=4.578, p<.001$. This result demonstrates that scores on the SIQ-Jr-2 were significantly lower than SIQ-Jr-1 scores. A $t$-test was also performed to compare the results between SIQ-Jr-1and SIQ-Jr-3. The results also demonstrated a statistically significant difference between SIQ-Jr-1 and SIQ-Jr-3, $t(60)=3.508, p<.001$. No significant differences were observed between the SIQ-Jr-2 and SIQ-Jr-3 scores. Results for all three administrations of the SIQ-Jr are presented in Figure 1.

\section{Figure 1. Mean SIQ-JR Scores Over the Course of Treatment}

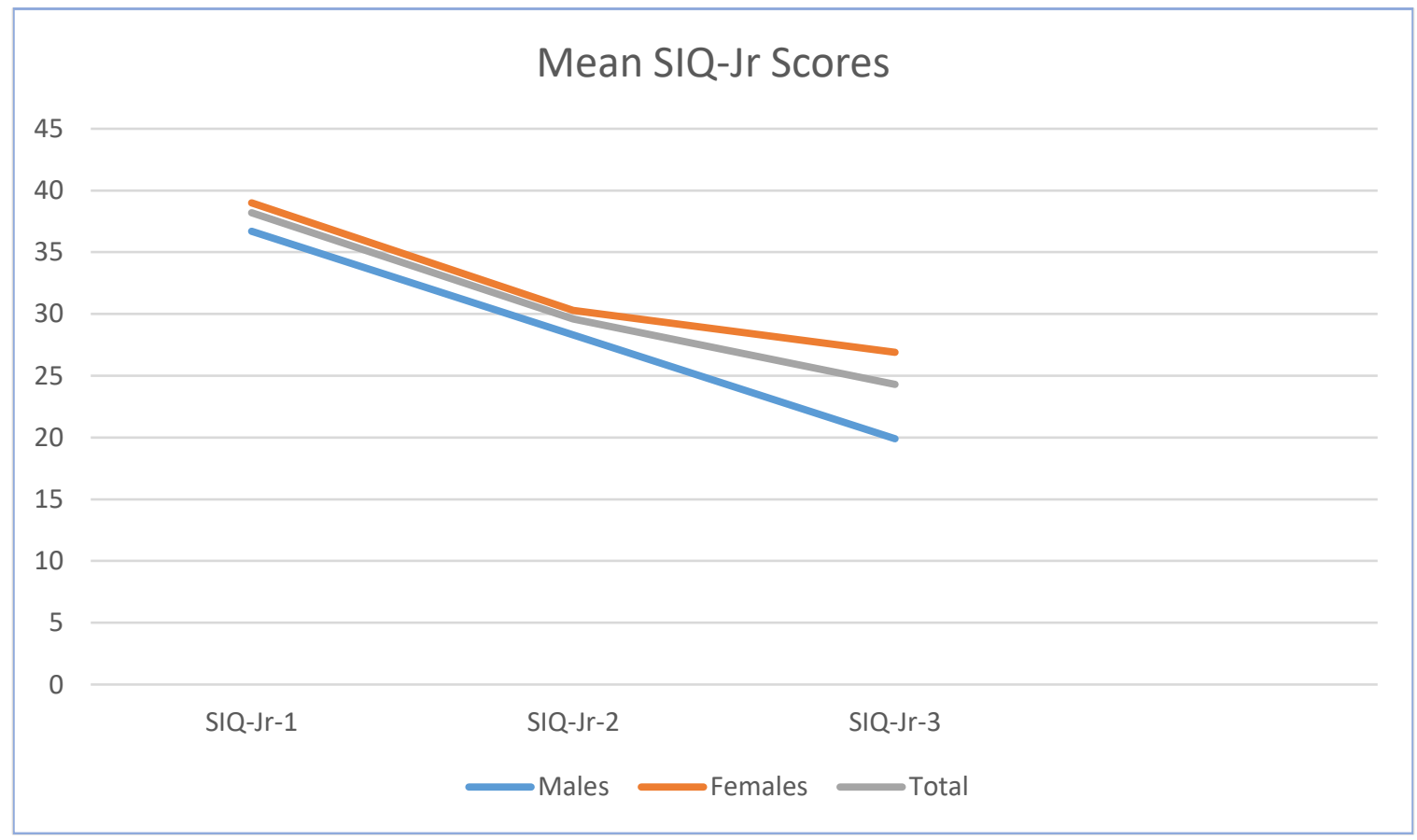


Table 1. SIQ-Jr Demographic and Mean Scores

\begin{tabular}{|c|c|c|c|c|c|c|c|c|c|c|c|c|}
\hline \multirow[b]{2}{*}{ Variable } & \multicolumn{4}{|c|}{ SIQ-Jr, Time 1} & \multicolumn{4}{|c|}{ SIQ-Jr, Time 2} & \multicolumn{4}{|c|}{ SIQ-Jr, Time 3} \\
\hline & $N$ & $\%$ & $\begin{array}{l}\text { Mean } \\
\text { score }\end{array}$ & $S D$ & $\boldsymbol{N}$ & $\%$ & $\begin{array}{l}\text { Mean } \\
\text { score }\end{array}$ & SD & $\boldsymbol{N}$ & $\%$ & $\begin{array}{l}\text { Mean } \\
\text { score }\end{array}$ & $S D$ \\
\hline & 93 & $100 \%$ & 38.2 & 21.6 & 78 & $84 \%$ & 29.6 & 17.9 & 43 & $46 \%$ & 24.3 & 17.6 \\
\hline \multicolumn{13}{|l|}{ Gender } \\
\hline Male & 34 & $37 \%$ & 36.7 & 20.6 & 28 & $36 \%$ & 28.3 & 19.7 & 16 & $37 \%$ & 19.9 & 16.7 \\
\hline Female & 59 & $63 \%$ & 39.0 & 22.3 & 50 & $64 \%$ & 30.3 & 16.9 & 27 & $63 \%$ & 26.9 & 17.9 \\
\hline Race/Ethnicity & 92* & $99 \%$ & 38.3 & 21.3 & 77* & $83 \%$ & 29.6 & 18.0 & 43 & $46 \%$ & 24.3 & 17.6 \\
\hline Caucasian & 54 & $59 \%$ & 37.1 & 21.3 & 46 & $60 \%$ & 27.7 & 17.1 & 24 & $56 \%$ & 21.4 & 19.6 \\
\hline Latino & 23 & $25 \%$ & 42.0 & 23.0 & 19 & $25 \%$ & 31.6 & 18.9 & 11 & $26 \%$ & 27.1 & 14.3 \\
\hline Other & 15 & $16 \%$ & 35.9 & 22.1 & 12 & $16 \%$ & 33.9 & 20.2 & 8 & $18 \%$ & 29.1 & 15.2 \\
\hline \multicolumn{13}{|l|}{ Age (years) } \\
\hline$\leq 12$ & 12 & $13 \%$ & 33.8 & 21.0 & 9 & $11 \%$ & 18.4 & 8.7 & 5 & $12 \%$ & 16.0 & 16.2 \\
\hline 13 & 19 & $20 \%$ & 34.6 & 22.3 & 18 & $23 \%$ & 27.8 & 19.0 & 11 & $26 \%$ & 22.6 & 15.8 \\
\hline 14 & 13 & $14 \%$ & 43.6 & 21.0 & 11 & $14 \%$ & 38.5 & 6.0 & 5 & $12 \%$ & 25.4 & 17.6 \\
\hline 15 & 17 & $18 \%$ & 35.2 & 22.4 & 12 & $15 \%$ & 28.7 & 16.9 & 8 & $19 \%$ & 29.0 & 20.5 \\
\hline 16 & 22 & $24 \%$ & 39.6 & 22.3 & 20 & $26 \%$ & 31.6 & 20.2 & 10 & $23 \%$ & 27.6 & 20.5 \\
\hline$\geq 17$ & 10 & $11 \%$ & 44.9 & 21.0 & 8 & $10 \%$ & 30.0 & 17.8 & 4 & $9 \%$ & 20.3 & 15.4 \\
\hline
\end{tabular}

Note. *Ethnicity data missing for one client who completed the SIQ-Jr at both Time 1 and Time 2. 


\section{Removing Barriers to Treatment for Suicidal Youth}

\section{Follow-Up Survey Results}

Follow-up survey results indicate that subsequent to the referral, 21 of the 99 clients had another suicide risk assessment, 11 required additional mental health treatment, and two were known to have attempted suicide. No SWF client in this sample went on to take his own life. Analyses of SIQ-Jr scores revealed significantly higher SIQ-Jr-3 scores for clients who were hospitalized at some point subsequent to SWF services, $F(1,11)=6.182, p<.05$.

\section{Discussion}

As the second leading cause of death in individuals aged 10 to 24 years (Heron, 2016), youth suicide is a major public health problem in the United States. Psychotherapeutic treatment can be effective in reducing suicidal behaviors, including suicide attempts (Ougrin et al., 2015). Unfortunately, many depressed and suicidal youth do not receive the mental health treatment they need (Cummings \& Druss, 2011; Freedenthal, 2007). Previous research has indicated a number of barriers to seeking mental health treatment, including not knowing where to get help and lack of transportation, barriers related to perceptions of mental health problems and treatment, and the financial cost of treatment (Larson et al., 2011, Gould et al., 2009; Kataoka, et al., 2007; Moscos et al., 2007; Owens et al., 2002). This study sought to examine the efficacy of providing free and accessible mental health treatment for suicidal youth.

Ninety-nine youth aged 8 to 18 years identified at risk for suicide were referred for treatment through SWF, a nonprofit organization that pays for counseling for suicidal youth. Ninety-three of the youth completed the SIQ-Jr at the beginning and end of treatment and referral sources completed a follow-up survey at the end of the year to examine treatment effectiveness.

Significantly more females were referred for SWF treatment than males. This is in line with previous studies that found suicidal females to be more likely to access mental health treatment than males (Farand et al., 2004; Guerra \& Vasiliadis, 2016). However, it is somewhat unexpected in that all SWF clients had undergone a suicide risk assessment at school prior to being referred and previous research has indicated that males and females are generally assessed for suicide risk at school at similar rates (Crepeau-Hobson, 2013).

Collectively, study results suggest that a model of service delivery such as SWF can be effective in reducing suicidal ideation and behaviors-both in the short-term and at follow-up. The vast majority of study clients had significantly reduced suicidal ideation and required no further 


\section{Removing Barriers to Treatment for Suicidal Youth}

intervention following treatment by a SWF mental health service provider. Previous research has indicated that barriers to treatment such as lack of adequate insurance, not knowing where to obtain help, and not having transportation can prevent suicidal youth from obtaining needed mental health treatment (Moscos et al., 2007). This study demonstrated that removing barriers to treatment can increase access and that the provision of psychotherapeutic counseling can reduce suicidal thoughts and behaviors in struggling youth.

Interestingly, study results indicated no significant age, gender, or racial/ethnic differences in levels of self-reported suicidal ideation. This is in contrast to national surveys (e.g., YRBS) that indicate that females and Hispanics are more likely to report seriously considering and attempting suicide in the previous 12 months than males and non-Hispanics (Kann et al., 2016). However, this may be due in part to differences in how suicidal ideation was assessed: the YRBS obtained this information via two individual survey questions whereas the present study utilized a more comprehensive measure of suicidal ideation (SIQ-Jr). Further, due to the relatively small sample size, the present study may have lacked adequate statistical power to detect ethnic differences. Regardless, the present study has clinical significance in that it provided evidence that the provision of mental health treatment can be effective in reducing suicidal ideation in males and females from both Hispanic and non-Hispanic backgrounds.

\section{Limitations}

There are several limitations to the current study that should be considered when reviewing these results. First, the sample was relatively small and included few members of some ethnic or racial groups, thus reducing the generalizability of the findings. The small numbers of youth who required additional intervention also limited more refined analysis into those factors related to need for treatment and support. In addition, although SWF therapists had been vetted to ensure expertise in terms of treating suicidal youth, their training, background, and theoretical orientations varied and treatment was not standardized across clients. Thus, it is not possible to drill down into identifying specific treatments/approaches that may be more effective with suicidal youth from different backgrounds and/or those with various risk factors. Future research should address these limitations to better ascertain effective means of reducing barriers and identify those psychotherapeutic treatments that may be most effective for youth who present with varying risk factors for suicide. 


\section{Removing Barriers to Treatment for Suicidal Youth}

\section{Implications for Practice}

Although a small number of study clients clearly needed more than what the 12 free sessions provided, the vast majority of these young people had significantly lower rates of suicidal ideation following treatment and most needed no additional intervention over the course of the following year. Participation in psychotherapeutic counseling following identification of suicide risk can be an effective means of suicide prevention. However, a program such as SWF could not be successful without both strong collaboration with school-based mental health professionals and effective school-based suicide risk assessment procedures in place. Such risk assessments help determine if a particular student is suicidal, and if so to what extent. These data are then used to guide interventions directed at meeting the student's needs (Miller, 2011), including referring for community-based treatment as appropriate. Research indicates that school-based risk assessments are effective in preventing suicide and assisting students in obtaining needed mental health support (Crepeau-Hobson, 2013). As such, risk assessment procedures should be implemented in all schools, including elementary schools as both previous research and the present study indicate that even elementary-age students can become suicidal. Consequently, developmentally appropriate risk assessment procedures must be in place at all grade levels.

The results of this study also have implications for training and professional development programs as school-based mental health professionals are critical players in assessing suicide risk. Having an understanding of evidence-based suicide prevention and risk assessment practices is key to saving lives in this context. School-based mental health professionals need to be trained to effectively assess suicide risk and to intervene as appropriate. Further, it is incumbent upon these individuals to identify community-based mental health providers who have the training and expertise to support suicidal youth and establish relationships with those individuals. Identification of free and/or affordable services (i.e., those available for free or at low cost; providers who accept Medicaid, etc.) is required to reduce barriers to treatment as these can impact the likelihood that families follow through when referrals are made (Allison et al., 2008). Finally, it is imperative that school-based mental health professionals follow up after a referral has been made to ensure follow through. Research indicates that this is a significant problem as approximately $12 \%$ to $36 \%$ of initial appointments are not attended (Carr, McDonnell, \& Owen, 1994; McKay, McCadam, \& Gonzales, 1996).

The present study provides some evidence that easily accessible, community-based mental health treatment can reduce suicidal thoughts and behaviors in youth. By removing social and 
Journal of Youth Development | http://jyd.pitt.edu/ | Vol. 14 Issue 3 DOI 10.5195/jyd.2019.731

Removing Barriers to Treatment for Suicidal Youth

financial barriers to treatment, programs such as the Second Wind Fund have the potential to save lives.

\section{References}

Allison, S., Roeger, L., \& Abbot, D. (2008). Overcoming barriers in referral from schools to mental health services. Australian Psychiatry, 16(1), 44-47.

Asarnow, J. R., Tompson, M. C., \& Berk, M. S. (2005). Adolescent depression: Family-focused treatment strategies. In W. Pinsof \& J. Lebow (Eds.) Family psychology: The art of the science (pp. 425450). New York, NY: Oxford University Press.

Carr, A., McDonnell, D., \& Owen, P. (1994). Audit and family systems consultation: Evaluation of practice at a child and family center. Journal of Family Therapy, 16, 143-157.

Centers for Disease Control. (2017). QuickStats: Suicide rates for teens aged 15-19 years, by sex United States, 1975-2015. Morbidity and Mortality Weekly Report (MMWR) 2017;66:816. doi:10.15585/mmwr.mm6630a6

Cherpitel, C. J., Borges, G. L., \& Wilcox, H. C. (2004). Acute alcohol use and suicidal behavior: A review of the literature. Alcoholism Clinical Exploratory Research, 28, 18S-28S. doi:10.1097/01.ALC.0000127411.61634.14

Chu, J.P., Hsieh, K., \& Tokars, D.A. (2011). Help-seeking tendencies in Asian Americans with suicidal ideation and attempts. Asian American Journal of Psychology, 2(1), 25-38. doi:10.1037/a0023326

Crepeau-Hobson, F. (2013). An exploratory study of suicide risk assessment practices in the school setting. Psychology in the Schools, 50(8), 810-822. doi:10.1002/pits.21705

Cummings, J. R., \& Druss, B. G. (2011). Racial/ethnic differences in mental health service use among adolescents with major depression. Journal of the American Academy of Child and Adolescent Psychiatry, 50(2), 160-170.

Farand, L., Renaud, J., \& Chagnon, F. (2004). Adolescent suicide in Quebec and prior utilization of medical service. Canadian Journal of Public Health, 95, 357-360.

Freedenthal, S. (2007). Racial disparities in mental health service use by adolescents who thought about or attempted suicide. Suicide and Life-Threatening Behavior, 371), 22-34.

Fried, L. E., Williams, S., Cabral, H., \& Hacker, K. (2012). Differences in risk factors for suicide attempts among $9^{\text {th }}$ and $11^{\text {th }}$ grade youth: A longitudinal perspective. The Journal of School Nursing, 29(2), $1-10$.

Gould, M. S., Marrocco, F. A., Hoagwood, K., Kleinman, M., Amakawa, L., \& Altschuler, E. (2009). Service use by at-risk youths after school-based suicide screening. Journal of the American Academy of Child and Adolescent Psychiatry, 48(12), 1193-1201. doi:10.1097/CHI.0b013e3181bef6d5 
Journal of Youth Development | http://jyd.pitt.edu/ | Vol. 14 Issue 3 DOI 10.5195/jyd.2019.731

\section{Removing Barriers to Treatment for Suicidal Youth}

Guerra, S. G., \& Vasiliadis, H. M. (2016). Gender differences in youth suicide and healthcare service use. Crisis, 374), 290-298.

Gutierrez, P. M., \& Osman, A. (2009). Getting the best return on your screening investment: An analysis of the Suicidal Ideation Questionnaire and Reynolds Adolescent Depression Scale. School Psychology Review, 38(2).

Heron, M. (2016). Deaths: Leading causes for 2014. National Vital Statistics Reports, 65(5). Retrieved from https://www.cdc.gov/nchs/data/nvsr/nvsr65/nvsr65_05.pdf

Husky, M., Sheridan, M., McGuire, L., \& Olfson, M. (2011). Mental health screening and follow-up care in public high schools. Journal of the American Academy of Child \& Adolescent Psychiatry, 50(9), 881-891. doi:10.1016/j.jaac.2011.05.013

Impey, M. M., \& Heun, R. R. (2012). Completed suicide, ideation and attempt in attention deficit hyperactivity disorder. Acta Psychiatrica Scandinavica, 125(2), 93-102

Kann, L., McManus, T., Harris W. A., Shanklin, S. L., Flint, K. H., Hawkins, J., . . . Zaza, S. (2016). Youth risk behavior surveillance - United States, 2015. MMWR Surveillance Summary 65(6), 1-174. Retrieved from https://www.cdc.gov/healthyyouth/data/yrbs/pdf/2015/ss6506_updated.pdf

Kataoka, S., Stein, B. D., Nadeem, E., \& Wong, M. (2007). Who gets care? Mental health service use following a school-based suicide prevention program. Journal of the American Academy of Child \& Adolescent Psychiatry, 46 (10), 1342-1348. doi:10.1097/chi.0b013e31813761fd

Kataoka, S. H., Zhang, L., \& Wells, K. B. (2002). Unmet need for mental health care among U.S. children: Variation by ethnicity and insurance status. The American Journal of Psychiatry, 159 (9), 15481555.

Kelly, T., Cornelius, J., \& Lynch, K. (2002). Psychiatric and substance use disorders as risk factors for attempted suicide among adolescents: A case control study. Suicide and Life Threatening Behavior, 32, 301-312. doi:10.1521/suli.32.3.301.22168.

Larson, J., dosReis, S., Stewart, M., Kushner, R., Frosch, E., \& Solomon, B. (2011). Barriers to mental health care for urban, lower income families referred from pediatric primary care. Administrative and Policy in Mental Health, 40, 159-167. doi:10.1007/s10488-011-0389-1

McKay, M. M., McCadam, K., \& Gonzales, J. J. (1996). Addressing the barriers to mental health services for inner city children and their caretakers. Community Mental Health Journal, 32, 353-361.

Michelmore, L. \& Hindley, P. (2012). Help-seeking for suicidal thoughts and self-harm in young people: A systematic review. Suicide and Life-Threatening Behavior, 42(5), 507-524. doi:10.1111/j.1943278X.2012.00108.x

Miller, D. N. (2011). Child and adolescent suicidal behavior: school-based prevention, assessment, and intervention. New York, NY: The Guilford Press.

Moskos, M. A., Olson, L., Halbern, S. R., \& Gray, D. (2007). Utah youth suicide study: Barriers to mental health treatment for adolescents. Suicide and Life-Threatening Behavior, 37(2), 179-186. 
Journal of Youth Development | http://jyd.pitt.edu/ | Vol. 14 Issue 3 DOI 10.5195/jyd.2019.731

\section{Removing Barriers to Treatment for Suicidal Youth}

Ougrin, D., Tranah T., Stahl, D., Moran, P., \& Asarnow, J. R. (2015). Therapeutic interventions for suicide attempts and self-harm in adolescents: Systematic review and meta-analysis. Journal of the American Academy of Child and Adolescent Psychiatry, 54(2):97-107.e2. doi:10.1016/j.jaac.2014.10.009

Owens, P. L., Hoagwood, K., Horwitz, S. M., Leaf, P. J., Poduska, J. M., Kellam, S. G., \& Ialongo, N. S. (2002). Barriers to children's mental health services. Journal of the American Academy of Child and Adolescent Psychiatry, 41(6), 731-738.

Reinecke, M. A., DuBois, D. L., \& Schultz, T. M. (2001). Social problem solving, mood, and suicidality among inpatient adolescents. Cognitive Therapy and Research, 25(6), 743-756.

Reynolds, W. M. (1987) Suicidal Ideation Questionnaire Professional Manual. Odessa, FL: Psychological Assessment Resources, Inc.

Verona, E., \& Shabnam, J. (2011). Dimensions of adolescent psychopathology and relationships to suicide risk indicators. Journal of Youth \& Adolescence, 40(8), 958-971.

Wang, P. S., Bergland, P. A., Olfson, M., \& Kessler, R. C. (2004). Delays in initial treatment contact after first onset of a mental disorder. Health Services Research 39(2), 393-416.

Wu, P., Katic, B.J., Liu, X., Fan, B., \& Fuller, C.J. (2010). Mental health service use among suicidal adolescents: Findings from a U.S. national community survey. Psychiatric Services, 61(1), 17-24. 